(2) Open Access Full Text Article

METHODOLOGY

\title{
A randomized, placebo-controlled, double-blind study to confirm the reversal of hepatorenal syndrome type I with terlipressin: the REVERSE trial design
}

\author{
This article was published in the following Dove Press journal: \\ Open Access Journal of Clinical Trials \\ 13 July 2012 \\ Number of times this article has been viewed
}

Background: Hepatorenal syndrome (HRS) is a rare disorder of marked renal dysfunction in patients with cirrhosis, ascites, and portal hypertension. Type 1 HRS is a rapidly progressive acute kidney injury that develops shortly after a precipitating event, followed by a deterioration of function of other organs (eg, heart, brain, liver, adrenal glands). Presently, no approved drug therapies exist for HRS type 1 in the USA, Canada, or Australia. Given the rarity of this condition and the existing unmet medical need for treatment, the US Food and Drug Administration granted orphan drug and fast-track designations for terlipressin. The objective of the REVERSE trial was to determine the efficacy and safety of intravenous terlipressin compared with placebo in the treatment of adults with HRS type 1 who were also receiving intravenous albumin.

Methods: 180 subjects with HRS type 1 were enrolled at 65 investigational sites located in the USA and ten sites in Canada. Patients were randomized in a 1:1 ratio to treatment with either intravenous terlipressin administered every 6 hours or placebo for up to 14 days. The primary efficacy measure was confirmed HRS reversal, defined as the percentage of patients with two serum creatinine values of $\leq 1.5 \mathrm{mg} / \mathrm{dL}$ at least 48 hours apart, on treatment, and without intervening renal replacement therapy or liver transplantation. Other efficacy measures included change in renal function as reflected in serum creatinine levels, fractional excretion of sodium, recurrence of HRS type 1, transplant-free, dialysis-free, and overall survival.

Discussion: Data from this pivotal study are intended to demonstrate whether terlipressin is effective in reversing HRS type 1, while providing the level of evidence necessary to define the risk-benefit profile of terlipressin.

Keywords: terlipressin, Lucassin, hepatorenal syndrome, REVERSE, renal dysfunction, critical care

\section{Introduction}

Hepatorenal syndrome (HRS) is a rare disorder of pronounced renal dysfunction in patients with end-stage liver disease. ${ }^{1,2}$ In the setting of liver cirrhosis, portal hypertension triggers the release of nitric oxide and other vasodilators, with resultant arterial vasodilatation in the splanchnic circulation and a decrease in systemic vascular resistance. ${ }^{1,3-5}$ In advanced cirrhosis, however, persistent and progressive arterial hypovolemia causes activation of vasoconstrictor mechanisms, including high plasma levels of renin activity, norepinephrine, and antidiuretic hormone, which lead to underperfusion of the kidneys. ${ }^{1,6,7}$
Correspondence: Khuramm Jamil Medical Director, Ikaria Lead, Terlipressin

53 Frontage Road, Third Floor Hampton, NJ 08827-900I USA

$\mathrm{Tel}+\mathrm{I} 9082386416$

Fax + I 9082386633

Email khurram.jamil@ikaria.com 
Diagnosis of HRS is based on the International Ascites Club (IAC) criteria. ${ }^{2}$ According to these criteria, HRS occurs in patients with end-stage cirrhosis and is marked by significantly impaired renal function in the absence of underlying renal pathology or systemic shock, and the lack of clinical response to volume expansion by albumin administration. ${ }^{2}$ The two identified types of HRS (type 1 and type 2) may be differentiated based on the rate of rise of serum creatinine (SCr). In HRS type 2, SCr rises gradually, and moderate renal impairment occurs ( $\mathrm{SCr}$ rises to $1.5-2.5 \mathrm{mg} / \mathrm{dL}$ ). ${ }^{2}$ In HRS type $1, \mathrm{SCr}$ doubles to $>2.5 \mathrm{mg} / \mathrm{dL}$ within 2 weeks, accompanied by rapid progressive renal impairment and a very poor prognosis, with $>80 \%$ mortality within 3 months. ${ }^{8-10}$ The estimated annual prevalence for HRS type 1 in the USA ranges from 9000 to 20,000 patients. ${ }^{8,10,11}$ Although liver transplantation offers a clear survival benefit, ${ }^{12}$ patients with HRS type 1 often die while waiting for organ availability. ${ }^{13,14}$ Therefore, a therapy to reverse HRS type 1 and provide a bridge to transplantation is desirable.

Terlipressin is a vasopressin analog approved for the treatment of HRS type 1 in France, the United Kingdom, Spain, Italy, Switzerland, and Denmark, as well as in several South American and Asian countries. In a number of clinical trials, terlipressin (1 to $2 \mathrm{mg}$; Glypressine, Ferring, Gentilly, France; Glypressin, Ferring, Madrid, Spain, and Langley, Berkshire, UK; Haemopressin SPC, Meduna Pharmaceuticals, Aschaffenburg, Germany), given as an intravenous bolus every 4 to 6 hours, has proven effective, marked by a significant increase in the rate of HRS type 1 reversal (eg, a decrease in SCr to $\leq 1.5 \mathrm{mg} / \mathrm{dL}$ without dialysis). ${ }^{15-18} \mathrm{~A}$ previous trial by Sanyal et $\mathrm{al}^{15}$ (also known as Study OT-0401) demonstrated that terlipressin was more effective than placebo for HRS reversal (34\% vs $13 \%, P=0.008)$ in patients with HRS type 1 . At present, there are no approved drug therapies for HRS type 1 in the USA, Australia, or Canada. Given the rare nature of this condition and the existing unmet medical need for treatment, orphan drug and fast-track designations were granted for terlipressin by the US Food and Drug Administration on October 29, 2004, and April 5, 2005, respectively.

In an attempt to verify the efficacy of terlipressin in HRS type 1 and address some limitations of the OT-0401study design, the Randomized, placEbo-controlled, doubleblind study to confirm the reVERSal of HRS type 1 with terliprEssin (REVERSE) trial was developed. The objective of this study was to determine the efficacy and safety of intravenous terlipressin compared with placebo in the treatment of adult patients with HRS type 1 receiving intravenous albumin. This report describes the rationale and design of the REVERSE trial, including details of patient inclusion/exclusion criteria, treatment protocol, primary efficacy and safety end points, and the statistical analyses plan.

\section{Materials and methods}

This pivotal, multicenter, Phase III, randomized, doubleblind, placebo-controlled, parallel-group study (US National Institutes of Health clinical trials identifier: NCT01143246) was expected to enroll 180 patients at 60-70 sites in the USA and five to ten sites in Canada. The protocol was approved by the institutional review board and/or independent ethics committee at each study site, and all study procedures were performed in accordance with good clinical practice. ${ }^{19}$ Written informed consent was obtained from each patient or a legally authorized representative prior to enrollment in the study.

\section{Patients}

Men and women aged 18 years or older having cirrhosis, ascites, and a diagnosis of HRS type 1 based on the 2007 IAC diagnostic criteria $^{2}$ were eligible for participation. HRS type 1 is commonly defined by a doubling of the initial SCr concentration to a level $>2.5 \mathrm{mg} / \mathrm{dL}$ in $<2$ weeks. The REVERSE trial enrolled patients with an SCr level $>2.5 \mathrm{mg} / \mathrm{dL}$ and either a doubling of $\mathrm{SCr}$ within 2 weeks or a change in $\mathrm{SCr}$ levels over time, indicating a trajectory with a slope equal to or greater than that of a doubling within 2 weeks. For ease of calculation, $\mathrm{SCr}$ trajectory was estimated by relating a defined

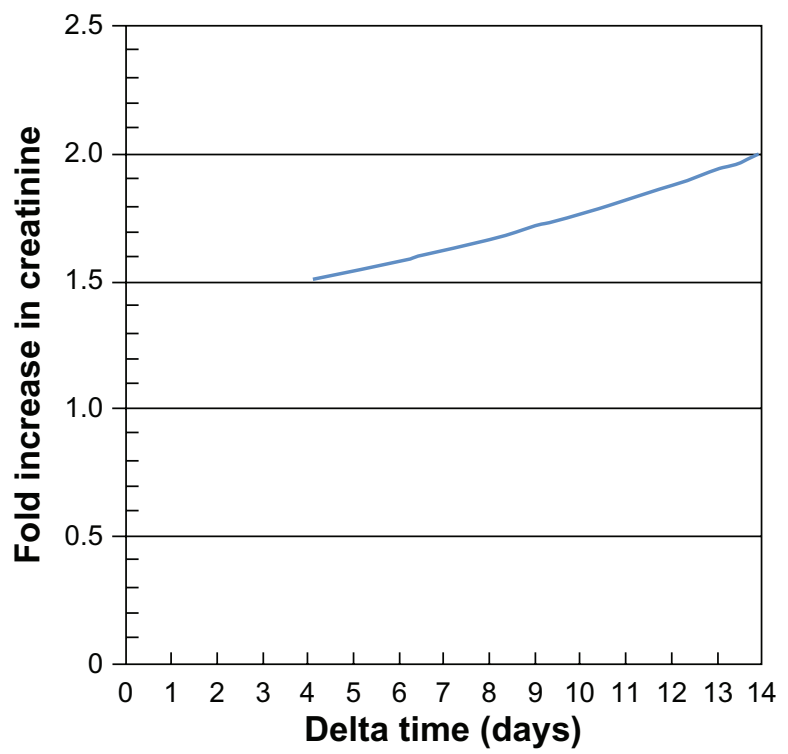

Figure I Nomogram to facilitate diagnosis of HRS type I for assessment of study eligibility.

Abbreviation: HRS, hepatorenal syndrome. 
"fold increase in $\mathrm{SCr}$ " to the elapsed time in days between two measured $\mathrm{SCr}$ values (Figure 1); greater proportional increases in $\mathrm{SCr}$ were required for observations of shorter duration. In situations where the time elapsed between $\mathrm{SCr}$ assessments was longer than 2 weeks, eligibility was determined on a case-by-case basis.

Exclusion criteria aimed to generate a patient sample limited to individuals with functional renal impairment secondary to cirrhosis and ascites, who could safely be administered terlipressin and who were deemed likely to survive through the active study period. Specifically, exclusion criteria included $\mathrm{SCr}>7 \mathrm{mg} / \mathrm{dL}$, shock, hypotension (mean arterial pressure $<70 \mathrm{~mm} \mathrm{Hg}$ or $>40 \mathrm{~mm} \mathrm{Hg}$ decrease in systolic blood pressure from baseline), or systemic inflammatory response syndrome, marked by two or more of the following: temperature $>38^{\circ} \mathrm{C}$ or $<36^{\circ} \mathrm{C}$; heart rate $>90$ beats/min; respiratory rate of $>20$ breaths/min or $\mathrm{PaCO}_{2}$ of $<32 \mathrm{~mm} \mathrm{Hg}$; or white blood cell count $>12,000 / \mu \mathrm{L}$ or $<4000 / \mu \mathrm{L}$ ) or sepsis (systemic inflammatory response syndrome with documented infection). Other exclusion criteria included $<2$ days of anti-infective therapy for documented or suspected infection; proteinuria $>500 \mathrm{mg} /$ day; hematuria or microhematuria; clinically significant cast on urinalysis; evidence of intrinsic or parenchymal renal disease; obstructive uropathy; other renal pathology on ultrasound or other medical imaging; recent (within 4 weeks) renal replacement therapy; recent treatment with nephrotoxic drugs such as aminoglycosides; more than three doses of nonsteroidal antiinflammatory drugs within the prior month or long-term use ( $\geq 2$ weeks) of orally administered neomycin; superimposed acute liver failure/injury due to factors other than alcoholic hepatitis (eg, acute viral hepatitis, recreational drug use, acetaminophen, toxins such as mushroom poisoning); or recent treatment ( $\leq 48$ hours) with octreotide, midodrine, vasopressin, dopamine, or other vasopressors. Patients with severe cardiovascular disease, life expectancy of $<3$ days, confirmed pregnancy, or known allergy to study medications or their components were also excluded.

\section{Study procedures and treatment regimens}

The parallel-group study design included a screening and pretreatment phase, an active study period, and a followup period (Figure 2). An overview of study assessments is presented in Table 1. Patients were qualified for study entry during screening; eligibility criteria were verified, prior medications documented, a diagnosis of HRS type 1 was established, and informed consent obtained. The

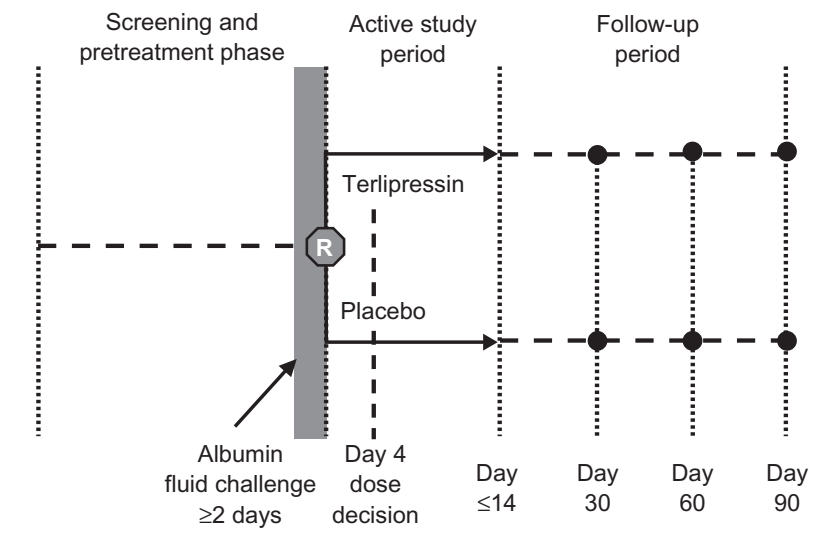

Figure 2 Overview of study design. Abbreviation: $\mathrm{R}$, randomization.

investigator at each study site screened patients for eligibility and reviewed clinical status daily throughout the active study period.

Qualified patients were enrolled in the study and entered the pretreatment period, during which baseline assessments and patient randomization occurred. Baseline assessments included a physical examination (including vital sign measurements), medical history, height and weight, ChildPugh score ${ }^{20}$ twelve-lead electrocardiogram, and drawing of blood samples for genetic marker and pharmacokinetic evaluations. Blood urea nitrogen, SCr, electrolytes, glucose, alkaline phosphatase, alanine amino transferase, aspartate amino transferase, protein, albumin, and bilirubin, along with urine creatinine and sodium, were measured at baseline and at protocol-specified time points throughout the active study period. In addition, glomerular filtration rate (GFR), international normalized ratio, encephalopathy score, ${ }^{21}$ model for end-stage liver disease (MELD) score, ${ }^{22}$ complete blood count, and differential and fractional excretion of sodium were calculated at baseline and at protocol-specified time points throughout the active study period. Baseline $\mathrm{SCr}$ (the qualifying SCr value) was obtained at least 48 hours after both diuretic withdrawal and the beginning of albumin fluid challenge, and no more than 8 hours prior to the start of study medication. As previously noted, this $\mathrm{SCr}$ value was required to be $\geq 2.25 \mathrm{mg} / \mathrm{dL}$ and at least $80 \%$ of the SCr value obtained during HRS diagnosis. Patients were stratified by qualifying $\mathrm{SCr}(<3.6 \mathrm{mg} / \mathrm{dL}$ or $\geq 3.6 \mathrm{mg} / \mathrm{dL})$ and the presence or absence of alcoholic hepatitis, and were randomized in a 1:1 ratio to receive either terlipressin or matching placebo. Treatment group assignments were made with randomization codes from a centralized interactive voice response system.

The active study period began with the first administration of terlipressin or matched placebo (Figure 3). 
Table I Overview of study assessments

\begin{tabular}{|c|c|c|c|c|c|c|c|}
\hline \multirow[t]{2}{*}{ Study assessment } & \multirow[t]{2}{*}{$\begin{array}{l}\text { Screening } \\
\text { period }\end{array}$} & \multicolumn{2}{|c|}{ Pretreatment period } & \multirow{2}{*}{$\begin{array}{l}\text { Active study } \\
\text { period } \\
\text { Days I-I4 } \\
\end{array}$} & \multicolumn{3}{|c|}{$\begin{array}{l}\text { Follow-up period } \\
\text { (Days from first dose) }\end{array}$} \\
\hline & & Study entry & $\begin{array}{l}\text { Baseline } \\
\text { assessment }\end{array}$ & & $\begin{array}{l}30 \text { days } \\
( \pm 7 \text { days })\end{array}$ & $\begin{array}{l}60 \text { days } \\
( \pm 14 \text { days })\end{array}$ & $\begin{array}{l}90 \text { days } \\
( \pm 14 \text { days })\end{array}$ \\
\hline Diagnosis of HRS type I established & $x$ & & & & & & \\
\hline Informed consent & $x$ & & & & & & \\
\hline Verification of study qualification & $x$ & & & & & & \\
\hline Randomization & & $x$ & & & & & \\
\hline Medical history & & & $x$ & & & & \\
\hline Prior medications & $x$ & $x$ & $x$ & & & & \\
\hline Concomitant medications $^{\mathrm{a}}$ & & & & $x$ & & & \\
\hline Physical examination & & & $x$ & & & & \\
\hline Weight & & & $x$ & & & & \\
\hline Height & & & $x$ & & & & \\
\hline 12-lead ECG & & & $x$ & & & & \\
\hline Child-Pugh score & & & $x$ & & & & \\
\hline Blood sample for genetic markers ${ }^{\mathrm{b}}$ & & & $x$ & & & & \\
\hline PK sampling & & & $x$ & $X^{c}$ & & & \\
\hline Study medication administration ${ }^{d}$ & & & $x$ & $<-------X----->>$ & & & \\
\hline Vital signs (BP, HR) ${ }^{e}$ & & & $x$ & <--------X------> & & & \\
\hline $\mathrm{SCr}$ and $\mathrm{BUN}^{\mathrm{f}}$ & & & $x$ & $<-------X----->>$ & & & \\
\hline Serum electrolytes ${ }^{g}$ & & & $x$ & $<-------X------>$ & & & \\
\hline GFR $^{g}$ & & & $x$ & $<-------X------>$ & & & \\
\hline Encephalopathy score ${ }^{g}$ & & & $x$ & $<-------X------>$ & & & \\
\hline $\begin{array}{l}\text { ALT, AST, ALP, protein, albumin, } \\
\text { bilirubin }^{h}\end{array}$ & & & $x$ & $<-------X------>$ & & & \\
\hline Serum glucose, calcium, magnesium ${ }^{h}$ & & & $x$ & <--------X------> & & & \\
\hline $\mathrm{INR}^{\mathrm{h}}$ & & & $x$ & $<-------X----->>$ & & & \\
\hline CBC and differential ${ }^{h}$ & & & $x$ & $<-------X------>$ & & & \\
\hline Spot urine creatinine and sodium ${ }^{h}$ & & & $x$ & $<-------X----->$ & & & \\
\hline Fractional excretion of sodium ${ }^{h}$ & & & $x$ & $<-------X------>$ & & & \\
\hline MELD score ${ }^{h}$ & & & $x$ & $<-------X------>$ & & & \\
\hline Nonserious adverse event' & & & & $<-------X------>$ & & & \\
\hline Serious adverse event $\mathrm{i}$ & & & & $<-------X------>$ & $<---X-->$ & & \\
\hline HRS type I recurrence assessment & & & & $<-------X------->$ & $\left.<-\mathrm{X}_{-}\right\rangle$ & & \\
\hline Mortality assessment & & & & 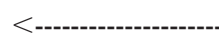 & ---------X- & --------------- & -----------> \\
\hline RRT assessment & & & & 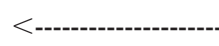 & -------X- & ---------------- & ----------> \\
\hline Transplantation assessment & & & & 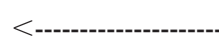 & ---------X- & --------------- & -----------> \\
\hline $\begin{array}{l}\text { Notes: }{ }^{a} \text { Concomitant medications include } \\
\text { minutes, } 30-120 \text { minutes, and } 3-5 \text { hours aft } \\
\text { pre-dose and } 5 \text { minutes, } 30 \text { minutes, and } \\
\text { during active treatment and until day } 14 \text { or } \\
\text { termination; 'monitored for up to } 7 \text { days af }\end{array}$ & $\begin{array}{l}\text { I albumin, IV sol } \\
\text { er study drug adr } \\
\text {, 2, and } 4 \text { hour } \\
\text { discharge, which } \\
\text { er end of treatm }\end{array}$ & $\begin{array}{l}\text { tions, and blood } P \\
\text { inistration; }{ }^{d} \text { maxim } \\
\text { after first dose, } t \\
\text { ever occurred first } \\
\text { ent; imonitored for }\end{array}$ & $\begin{array}{l}\text { oducts; boptional; } \\
\text { Im duration of tre } \\
\text { en at pre-dose, } 5 \\
\text { gassessed once da } \\
\text { ip to } 30 \text { days afte }\end{array}$ & $\begin{array}{l}\text { PK samples were take } \\
\text { ment was } 16 \text { days if } \mathrm{HI} \\
\text { ninutes, and } 2 \text { hours } \\
\text { during treatment day } \\
\text { end of treatment. }\end{array}$ & $\begin{array}{l}\text { during the fir } \\
\text { S reversal was } \\
\text { fter each subs } \\
\text {; hassessed on }\end{array}$ & $\begin{array}{l}\text { dose interval o } \\
\text { rst achieved on } \\
\text { quent dose; fasse } \\
\text { ays I, } 3 \text {, and } 7 \text {, a }\end{array}$ & $\begin{array}{l}\text { day I at } 5-10 \\
\text { ay } 14 \text {; eassessed } \\
\text { sed once daily } \\
\text { Id at treatment }\end{array}$ \\
\hline $\begin{array}{l}\text { Abbreviations: ALP, alkaline phosphatase } \\
\text { blood count; ECG, electrocardiogram; GFP } \\
\text { stage liver disease; PK, pharmacokinetic; RF }\end{array}$ & ALT, alanine am & notransferase; AST & ate; HRS, hepat & $\begin{array}{l}\text { nsferase; BP, blood pre } \\
\text { enal syndrome; INR, i }\end{array}$ & ure; BUN, bl & urea nitrogen; & $\begin{array}{l}\mathrm{CBC} \text {, complete } \\
\mathrm{D} \text {, model end- }\end{array}$ \\
\hline
\end{tabular}

During this period, treatment with the blinded study drug continued until at least two $\mathrm{SCr}$ values $\leq 1.5 \mathrm{mg} / \mathrm{dL}$ were obtained at least 48 hours apart, or up to 14 days. Duration of treatment was allowed to extend to a maximum of 15 or 16 days if HRS reversal was first achieved on days 13 or 14 , respectively.

Patients in the active treatment group received terlipressin $1 \mathrm{mg}$ given intravenously every 6 hours as a slow bolus injection over 2 minutes. The criteria for dose increases, study discontinuation, treatment resumption, and treatment completion during the active study period are shown in Figure 3. The dosing regimen for patients in the placebo (6 mL lyophilized mannitol solution) group was identical to the terlipressin regimen. Patients and all study personnel remained blinded to treatments throughout the study unless unblinding was required by a medical emergency. Administration of albumin at doses of 20 to 40 grams/ day following the albumin challenge was recommended, 

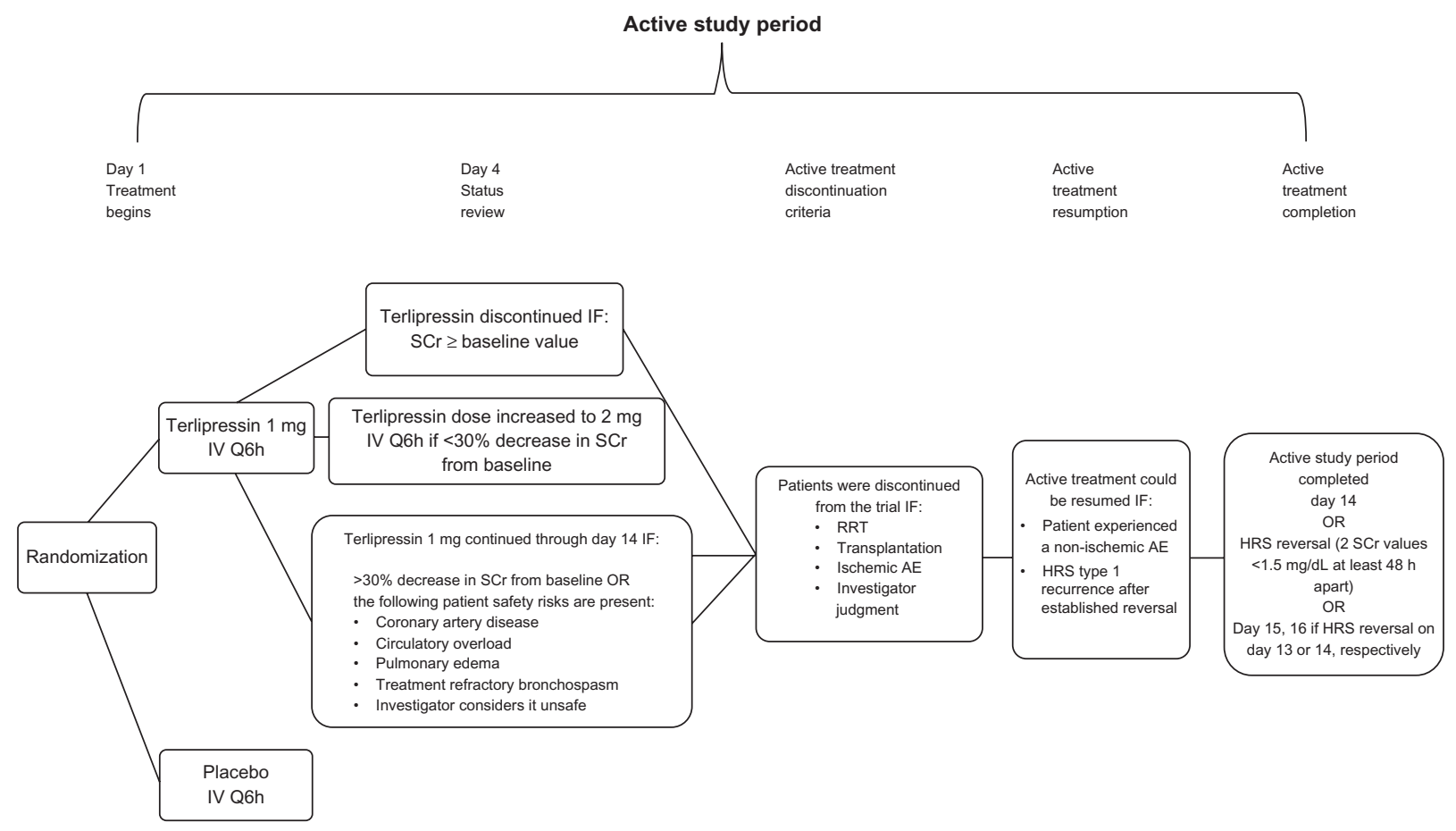

Figure 3 Algorithm: active study period treatment.

Abbreviations: AE, adverse event; HRS, hepatorenal syndrome; IV, intravenous; RRT, renal replacement therapy; SCr, serum creatinine.

as clinically indicated, for all patients in both study arms as per current IAC guidelines. ${ }^{2}$ The concomitant use of vasopressive drugs (ie, midodrine, vasopressin, dopamine, dobutamine, norepinephrine), octreotide, prostaglandin analogs (eg, misoprostol), and nonsteroidal anti-inflammatory drugs was prohibited during the study. The use of diuretics was discouraged, unless such use was medically required for fluid overload.

The follow-up period began after the end of study treatment and concluded 90 days after the start of study treatment. Patients were contacted $30( \pm 7), 60( \pm 14)$, and $90( \pm 14)$ days after the first day of study treatment for assessment of survival, renal replacement therapy, and transplantation. No clinical laboratory tests were scheduled to occur during the follow-up period. Patients had the right to discontinue treatment and/or participation in the study at any time, and the investigator could have discontinued any patient at any time for any reason. Patients who discontinued treatment due to an adverse event (AE) were followed until the event resolved or stabilized.

\section{Outcome assessments}

\section{Efficacy outcome measures}

All patients had SCr assessed at baseline and daily throughout the active study period or upon early study discontinuation. The primary efficacy measure was the percentage of patients with confirmed HRS reversal. Criteria for defining patients with HRS reversal are shown in Figure 4. The date and time of the first observed $\mathrm{SCr}$ value of $\leq 1.5 \mathrm{mg} / \mathrm{dL}$ on treatment were used for calculating the time window for the confirmatory $\mathrm{SCr}$ value. The first $\mathrm{SCr}$ value of $\leq 1.5 \mathrm{mg} / \mathrm{dL}$ occurring during the time window for confirmation was selected as the second and confirmatory value. Any SCr values obtained after liver transplantation or renal replacement therapy were excluded from efficacy analyses.

Other efficacy measures included change in renal function from baseline through the end of treatment (up to 24 hours after the last dose of study medication) as reflected in daily $\mathrm{SCr}$ values; incidence of HRS reversal, defined as at least one $\mathrm{SCr}$ value of $\leq 1.5 \mathrm{mg} / \mathrm{dL}$ while on treatment (up to 24 hours after last dose); transplant-free survival through 90 days after randomization; overall survival through 90 days after randomization; change in GFR from baseline through end of treatment calculated using both the Modification of Diet and Renal Disease and the Cockcroft-Gault equations; change from baseline through end of treatment in fractional excretion of sodium; incidence of HRS type 1 recurrence, defined as an $\mathrm{SCr}$ value of $\geq 2.5 \mathrm{mg} / \mathrm{dL}$ in the absence of other causes of renal impairment occurring until transplantation; day of discharge from the study site, or day 14, whichever occurred first; and dialysis-free survival through 90 days after randomization. 


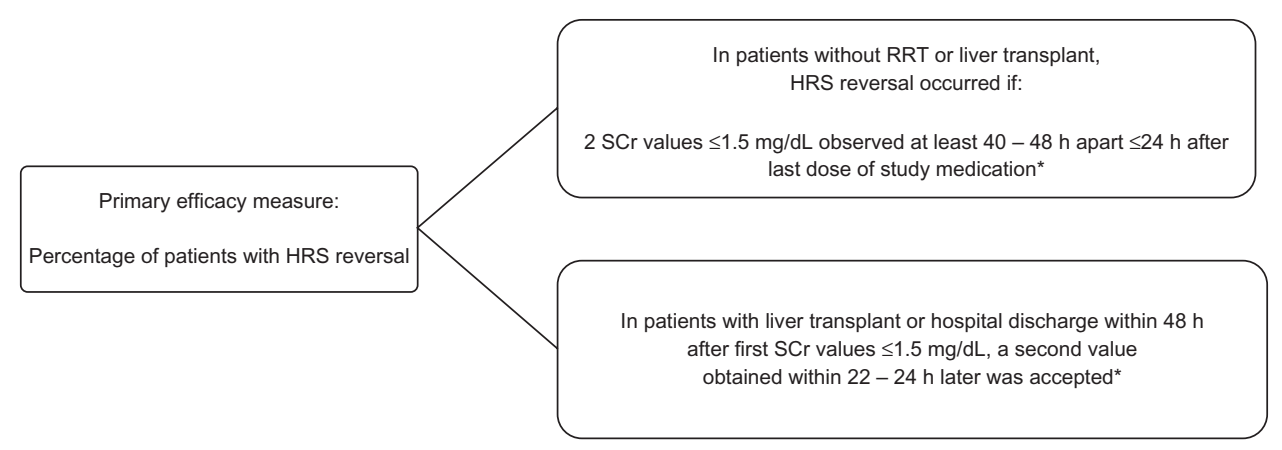

Figure 4 Efficacy analysis criteria summary.

Note: $*$ The $\mathrm{SCr}$ values $\leq 1.5 \mathrm{mg} / \mathrm{dL}$ did not have to be sequential, but intervening $\mathrm{SCr}$ values could not be $\geq 1.8 \mathrm{mg} / \mathrm{dL}$.

Abbreviations: HRS, hepatorenal syndrome; RRT, renal replacement therapy; $\mathrm{SCr}$, serum creatinine.

\section{Exploratory outcome assessments}

Exploratory subgroup analyses were also performed. These included time to transplantation of up to 90 days, summarized for patients who underwent transplantation; transplant-free survival and overall survival stratified by responders versus nonresponders for the HRS reversal and confirmed HRS reversal outcomes; and evaluation of efficacy outcomes stratified by baseline prognostic factors.

Blood samples were obtained from all patients, if possible, to characterize the pharmacokinetics of terlipressin and its metabolite lysine-vasopressin. In consideration of the patients' condition, a sparse sampling approach was used to collect blood samples from each patient. Pharmacokinetic analyses were conducted using nonlinear mixed-effects modeling.

All patients were invited to participate in a biomarker genetic evaluation, subject to institutional review board and/ or independent ethics committee approval. Blood samples were obtained, on a voluntary basis, for analyses regarding potential biomarkers that might reflect treatment response, hemodynamic effects, or the risk-benefit profile associated with terlipressin treatment. The plans for genetic evaluations included, but were not limited to, single nucleotide polymorphisms in the promoter region of the vasopressin $\mathrm{V} 1$ receptor gene and genome-wide association scans to study genetic variation at other single nucleotide polymorphism levels. The total additional volume of blood loss for biomarker genetic assessments was approximately $10 \mathrm{~mL}$ per patient.

\section{Safety outcome measures}

Safety outcome measures included vital signs, encephalopathy score, MELD score, and AEs. Vital signs (heart rate and blood pressure) were assessed at baseline, before administration of each dose of the study drug, at 5 minutes, 30 minutes, and 1,2, and 4 hours after the first dose of the study drug, and at 5 minutes and 2 hours after all other doses of the study drug. Encephalopathy was assessed and graded according to the West Haven Criteria for Semiquantitative Grading of Mental State (Table 2). ${ }^{21}$ MELD (which incorporates an international normalized ratio, $\mathrm{SCr}$, and serum bilirubin) is a validated model for prediction of survival in patients with liver diseases. ${ }^{22}$ MELD scores were calculated at baseline and on treatment days 1,3 , and 7 , and on the day of treatment termination. AEs were assessed and recorded beginning with the first administration of the study drug and continuing until 7 days after the study drug was discontinued. An AE was defined as any unfavorable and unintended sign, symptom, or disease temporally associated with the use of a medicinal product during the study, whether or not the sign, symptom, or disease was considered related to the medicinal product. Serious AEs were assessed and recorded until 30 days after the study drug was discontinued. A serious AE was defined as any AE that was life-threatening or resulted in death, resulted in persistent or significant disability, required or prolonged hospitalization, was a congenital anomaly or birth defect, or

Table 2 West Haven criteria for semiquantitative grading of mental state ${ }^{21}$

\begin{tabular}{|c|c|}
\hline \multirow[t]{4}{*}{ Grade I } & Trivial lack of awareness \\
\hline & Euphoria or anxiety \\
\hline & Shortened attention span \\
\hline & Impaired performance of addition \\
\hline \multirow[t]{5}{*}{ Grade 2} & Lethargy or apathy \\
\hline & Minimal disorientation for time or place \\
\hline & Subtle personality change \\
\hline & Inappropriate behavior \\
\hline & Impaired performance of subtraction \\
\hline \multirow[t]{4}{*}{ Grade 3} & Somnolence to semi-stupor, but responsive \\
\hline & to verbal stimuli \\
\hline & Confusion \\
\hline & Gross disorientation \\
\hline Grade 4 & Coma (unresponsive to verbal or noxious stimuli) \\
\hline
\end{tabular}


was another medically significant event that might jeopardize the patient and might require medical or surgical intervention. Deaths were reported up to 90 days after initiation of the study drug.

\section{Statistical analysis}

The estimate of sample size was based on data from the OT-0401 study reported by Sanyal and colleagues, ${ }^{15}$ in which the rate of confirmed HRS reversal for patients with a baseline $\mathrm{SCr}$ value of $\leq 7 \mathrm{mg} / \mathrm{dL}$ was $12.5 \%$ (7/56) in the placebo group and 36\% (18/50) in the group treated with terlipressin. An estimated enrollment of 180 patients (90 in each treatment group) was needed to provide $93 \%$ power for the primary efficacy analysis to detect a statistically significant difference ( $P \leq 0.05$ using a two-sided test) between the two treatment groups. All statistical analyses were performed using SAS software version 9.1.3 or higher (SAS Institute Inc, Cary, NC, USA) with a two-sided significance level of 0.05 , unless otherwise noted. If enrollment reached 180 patients with fewer than 30 events of confirmed HRS reversal being observed, enrollment continued until at least 30 events were observed.

The efficacy population, defined as all randomized patients who had had at least one baseline assessment, was used for all efficacy analyses. The safety population included all randomized patients who received at least one dose of the study drug. Treatment classification was based on randomized treatment for all efficacy analyses and on actual treatment received for all safety analyses.

Between-group differences were analyzed using an analysis of variance model with treatment as a factor. Categorical variables were summarized by frequency and percent; a Cochran-Mantel-Haenszel chi-square test was used to assess between-group differences.

The primary efficacy analysis data for the two treatment groups were compared using the Cochran-Mantel-Haenszel chi-square test stratified by qualifying $\mathrm{SCr}(<3.6 \mathrm{mg} / \mathrm{dL}$ or $\geq 3.6 \mathrm{mg} / \mathrm{dL}$ ) and the presence/absence of alcoholic hepatitis. If the proportion of patients with treatment success was small ( $<5$ events per stratified category cell), an unstratified chi-square test was used instead of the CochranMantel-Haenszel test. If the number of events per cell was still $<5$, then Fisher's exact test was used. If a significant difference between treatment groups was observed in the primary efficacy analysis, an analysis of secondary efficacy measures was conducted in sequence as follows: (1) incidence of HRS reversal (defined as at least one $\mathrm{SCr} \leq 1.5 \mathrm{mg} / \mathrm{dL}$ on treatment) was analyzed in the same manner as the primary outcome measure; (2) transplant-free survival up to 90 days was analyzed using a two-sample log rank test stratified by qualifying $\mathrm{SCr}$ and the presence/absence of alcoholic hepatitis; and (3) overall survival at 90 days was analyzed using a two-sample log rank test stratified by qualifying $\mathrm{SCr}$ and presence/absence of alcoholic hepatitis. A significance level of 0.05 was used for analysis of secondary outcome measures, and testing was stopped if the between-group difference was not significant for a secondary outcome measure.

Analyses of other efficacy outcome measures were conducted as follows: change from baseline through end of treatment in renal function ( $\mathrm{SCr}$ ) was analyzed using repeated measures analysis of covariance, with treatment as a main effect, and baseline qualifying SCr and day as covariates. HRS type 1 recurrence until transplantation, hospital discharge, or day 14 was summarized using descriptive statistics (number [n], frequency, and percentages). Continuous variables such as GFR and fractional excretion of sodium were analyzed by repeated measures of covariance, with treatment as a main effect and day and strata as covariates. Finally, dialysis-free survival up to 90 days was analyzed using a two-sample log rank test stratified by qualifying $\mathrm{SCr}$ and the presence/ absence of alcoholic hepatitis.

AEs were summarized by frequency and percent; Fisher's exact test was used to analyze between-group differences in the frequency of AEs. Vital signs (systolic blood pressure, diastolic blood pressure, heart rate, and mean arterial pressure) were summarized as individual values, daily averages, and maximum and minimum values. Change from baseline in encephalopathy score, MELD score, and serum urea nitrogen were analyzed similarly to the analysis of change from baseline in renal function. Other continuous safety variables (eg, labs) were analyzed using an analysis of variance on change from baseline, with treatment as a main effect, and with qualifying $\mathrm{SCr}$ and the presence/absence of alcoholic hepatitis as blocking factors. Other categorical safety variables (eg, lab shifts) were analyzed using the Cochran-Mantel-Haenszel test stratified by qualifying $\mathrm{SCr}$ and the presence/absence of alcoholic hepatitis.

\section{Discussion}

HRS type 1 is a serious disorder, complicating decompensated chronic liver disease with cirrhosis; the optimal treatment for the underlying cause of HRS type 1 is liver transplantation. ${ }^{23}$ However, many patients will not survive long enough to receive a liver transplant. Therapy that provides a bridge to transplantation is desperately needed. Pretransplant renal impairment is associated with increased 
post-transplant complications and a decrease in the net benefit of liver transplantation. ${ }^{15,24-27}$ Conversely, HRS reversal is associated with improved overall survival, ${ }^{15}$ and therapy that reverses HRS type 1 appears likely to be associated with improved post-transplant outcomes. ${ }^{24,28}$ In those patients who are not candidates for liver transplantation, HRS reversal facilitates medical management and may provide the survival time needed for improvement in underlying liver disease (eg, alcoholic hepatitis). At present, there are no approved drug therapies for HRS type 1 in the USA, Canada, or Australia.

Increased understanding of the pathophysiology of HRS type 1 has demonstrated that vasoconstrictive drug therapy may reverse HRS type $1 ;^{29,30}$ while many vasoconstrictive agents have been investigated, only terlipressin has demonstrated level 1A evidence in reversing HRS type $1 .^{15,18}$ Terlipressin has been the most widely used vasoconstrictor agent in HRS type 1. Terlipressin is a vasopressin analog derived from the natural hormone lysine-vasopressin. Vasopressin and its analogs interact with both V1 and V2 receptors. V1 receptors are located in vascular smooth muscle and are responsible for vasoconstriction when stimulated. V1 receptors are also located in other smooth muscle, such as the uterus, bladder, and gastrointestinal tract. In circumstances of catecholamine-resistant shock, these receptors have been demonstrated to be responsible for vasoconstriction. ${ }^{31}$ The postulated mechanism of action for this effect is that the stimulation of $\mathrm{V} 1$ receptors in vascular smooth muscle leads to an increase in cytoplasmic ionized calcium via the phosphatidylinositol biphosphate cascade. ${ }^{31-33}$ In HRS type 1, the effect that terlipressin promulgates is mediated by vasoconstriction of the splanchnic arterial blood vessels reducing blood flow to the system, thereby providing indirect volume expansion. ${ }^{3}$ Stimulation of V2 receptors responsible for an antidiuretic effect is less desirable in HRS. When comparing the selectivity of vasopressin and terlipressin, terlipressin has been found to have 2.2 times greater selectivity than vasopressin at the $\mathrm{V} 1$ receptor. ${ }^{34}$

In addition to its relative pharmacodynamic selectivity, terlipressin offers a pharmacokinetic advantage that makes it better for the treatment of HRS type 1. Terlipressin is a synthetic 12-amino-acid protein that is similar to endogenous human vasopressin except for the substitution of lysine for arginine at the eighth position of the endogenous molecule and the addition of three glycyl residues at the amino terminus. The duration of action of terlipressin is longer than that of vasopressin and is due to the stepwise cleavage of the N-terminal glycyl residues of terlipressin by various tissue peptidases, resulting in release of the pharmacologically active metabolite lysine-vasopressin, which has $>95 \%$ of the activity of the drug. ${ }^{35}$ It is for this reason terlipressin may be considered a prodrug. Once formed, lysine-vasopressin is eliminated by plasma peptidase losing its vasopressinergic activity. ${ }^{36,37}$ There is a biphasic decline in plasma terlipressin concentration, with an elimination half-life of 50 minutes. Terlipressin administration results in a marked increase in plasma concentrations of lysine-vasopressin, which peaks at 60-120 minutes, and remains elevated for at least $180-240$ minutes. ${ }^{37}$ In comparison, vasopressin is rapidly destroyed by a similar mechanism, with a half-life of 6-20 minutes. ${ }^{37}$ The extended effect of terlipressin allows for intermittent dosing and a more reliable clinical effect in HRS type 1.

A previous trial of terlipressin in patients with HRS type 1, OT-0401, provided important insight into the potential efficacy of terlipressin in HRS type 1 patients. ${ }^{15}$ However, additional important criteria needed to be evaluated, and the REVERSE trial was designed to evaluate these criteria. In order to ensure the best opportunity to demonstrate clinical success and advance HRS type 1 therapy, a number of incremental changes in study design have been made, based on discoveries from the previous trial, where the measurement of renal function was assessed at the end of 14 days of therapy. In the REVERSE trial, assessment of HRS reversal was based on the percentage of patients with two $\mathrm{SCr}$ values $\leq 1.5 \mathrm{mg} / \mathrm{dL}$. This brings the definition of HRS reversal more in line with the standard accepted in the medical community. ${ }^{38,39}$

Another important change to the REVERSE trial, as compared with Study OT-0401, is the method of using an $\mathrm{SCr}$ rate-of-rise nomogram in the inclusion criteria. In Study OT-0401, patients had to have an $\mathrm{SCr}$ value of $\geq 2.5 \mathrm{mg} / \mathrm{dL}$ and a doubling of $\mathrm{SCr}$ within 2 weeks. This 2 -week time requirement for assessment poses difficulty in the enrollment time frame, because HRS is a critical illness with a median survival time of 2-4 weeks. Therefore, a solution to a shorter duration of observation is necessary. The OT-0401 protocol permitted enrollment of patients with a change in SCr levels over time, resulting in a trajectory with a slope equal to or greater than that of a doubling within 2 weeks. Nevertheless, given the known daily fluctuations in $\mathrm{SCr}$ as a marker of GFR, an estimated SCr trajectory over a short period could over- or underestimate changes in renal function. In the REVERSE trial, to provide more consistent assessment for renal function at the time of enrollment, screening period estimates of $\mathrm{SCr}$ increases over time were based on a nomogram, which included both the time for an increase in SCr and the absolute 
$\mathrm{SCr}$ value. The nomogram was developed based on a slopecriteria regression analysis for proportional increases likely to be representative of at least doubling within 2 weeks. To our knowledge, REVERSE was the first study to employ such a nomogram.

In the REVERSE trial, the screening period ensured that patients truly had HRS type 1 that was not reversed by a fluid challenge. In order to ensure the accuracy of the diagnosis, albumin was administered according to IAC guidelines, with consistent daily administrations when clinically feasible. ${ }^{2}$ Further, unlike in Study OT-0401, the qualifying SCr value in the REVERSE trial had to be at least $80 \%$ of the diagnostic (pre-fluid challenge) SCr value. This ensured that subjects who were likely to respond to continued fluid support with albumin alone were not included. This increased the robustness of the trial by allowing for the measurement of efficacy achieved with terlipressin over subjects who responded to albumin.

Finally, in highlighting the major differences between the two trials, the REVERSE trial excluded patients with exceedingly high baseline SCr levels ( $\geq 7.0 \mathrm{mg} / \mathrm{dL}$ ). This decision was based on the fact that none of these patients in Study OT-0401 responded to terlipressin. ${ }^{15}$ Additionally, data suggest that such high $\mathrm{SCr}$ levels have a negative predictive value when determining response rates. ${ }^{40,41}$ This is likely a reflection of the extent of advanced HRS type 1; such patients are unlikely to respond to any therapy short of a combined liver and kidney transplant. Another possibility lending to the different characteristics in response rates includes the pharmacogenomic impact, which to our knowledge has not been examined in previous trials. Therefore, in the REVERSE trial, samples were collected in subjects who provided additional consent. ${ }^{42,43}$

\section{Conclusion}

Four randomized, controlled trials of terlipressin in HRS type $1,15,16,18,30$ along with recent meta-analyses of these and other trials, support the role of terlipressin in the treatment of HRS type $1 .^{29,39}$ The largest of the randomized, placebocontrolled trials studied 112 patients with HRS type 1 who were treated with terlipressin or placebo. ${ }^{15}$ The REVERSE trial represents the final Phase III confirmatory trial. Overall, data from this pivotal study and the existing literature will provide the level of evidence necessary to define the risk-benefit profile of terlipressin for use in patients with HRS type 1 .

\section{Description of authors' roles}

Study conception and design were the work of TD Boyer, JJ Medicis, J Potenziano, C Pappas, and K Jamil. The study investigator was TD Boyer. Collection and assembly of data were done by TD Boyer, JJ Medicis, J Potenziano, C Pappas, and K Jamil. Data analysis and interpretation were conducted by TD Boyer, JJ Medicis, J Potenziano, C Pappas, and K Jamil. Manuscript preparation was done by TD Boyer, JJ Medicis, J Potenziano, C Pappas, and K Jamil. Manuscript review and revisions were done by TD. Boyer, JJ Medicis, J Potenziano, C Pappas, and K Jamil. Final approval of manuscript was done by TD Boyer, JJ Medicis, J Potenziano, C Pappas, and K Jamil.

\section{Acknowledgments}

This study was sponsored by Ikaria, Inc. The authors thank Peter Teuber and Candice Teuber of Orphan Therapeutics for assistance with study design. The authors also thank Michael Morren, $\mathrm{RPh}$, of Peloton Advantage, LLC, for providing editorial assistance during manuscript preparation, which was funded by Ikaria, Inc.

The authors gratefully acknowledge the participation of the following study sites (all in the USA, except where noted): University of Arizona, Tucson, AZ; Virginia Commonwealth University, Richmond, VA; New York University Medical Center, New York, NY; Bellevue Hospital Center, New York, NY; Temple University Hospital, Philadelphia, PA; Indiana University, Indianapolis, IN; Hunter Holmes McGuire VA Medical Center, Richmond, VA; Henry Ford Health System, Detroit, MI; University of Colorado Denver, Aurora, CO; St Luke's Episcopal HospitalBaylor College of Medicine, Houston, TX; University of California, San Diego and Southern California Liver Centers, Coronado, CA; Mercy Medical Center, Baltimore, MD; Northwestern University, Chicago, IL; Georgetown University Hospital, Washington, DC; University of Pennsylvania, Philadelphia, PA; University of Texas Southwestern Medical Center, Dallas, TX; Parkland Hospital, Dallas, TX; Albert Einstein Medical Center, Philadelphia, PA; California Pacific Medical Center, San Francisco, CA; University of California, Sacramento, CA; Baylor University Medical Center, Dallas, TX; VA San Diego Healthcare System and University of California, San Diego, CA; Mt Sinai Medical Center, New York, NY; Emory University, Atlanta, GA; Washington University, St Louis, MO; USC Healthcare, Los Angeles, CA; Loma Linda University Medical Center, Loma Linda, CA; Toronto General Hospital, Toronto, Ontario, Canada; University of Miami, Miami, FL; Tulane University Health Sciences Center, New Orleans, LA; Yale University School of Medicine, New Haven, CT; Scripps Green Hospital, La Jolla, CA; University of Texas Medical Branch, Galveston, 
TX; University of Minnesota, Minneapolis, MN; Mayo Clinic, Jacksonville, FL; Oregon Health and Science University, Portland, OR; University of Nebraska Medical Center, Omaha, NE; Vanderbilt Medical Center, Nashville, TN; Saint Louis University, St Louis, MO; University of Chicago, Chicago, IL; Lahey Clinic Medical Center, Burlington, MA; University of Cincinnati, Cincinnati, OH; Mayo Clinic, Phoenix, AZ; Beth Israel Deaconess Medical Center, Boston, MA; University of Wisconsin School of Medicine and Public Health, Madison, WI; Virginia Mason Medical Center, Seattle, WA; University of Utah, Salt Lake City, UT; Cleveland Clinic, Cleveland, OH; Centre Hospitalier De L'Universite De Montreal, Montreal, Quebec, Canada; Texas Transplant Institute, San Antonio, TX; Rush University Medical Center, Chicago, IL; University of California, San Francisco, CA; University of Kentucky, Lexington, KY; Methodist Center for Liver Disease and Transplantation, Houston, TX; Westchester Medical Center, Valhalla, NY; University of Maryland Baltimore, Baltimore, MD; The Ohio State University, Columbus, OH; Saint Luke's Hospital, Kansas City, MO; Iowa City VA Healthcare System, Iowa City, IA; Carolinas Medical Center, Charlotte, NC; INTEGRIS Baptist Medical Center, Inc, Oklahoma City, OK; University of Massachusetts Memorial Medical Center, Worcester, MA; University of Texas-Houston, Houston, TX; Hartford Hospital, Hartford, CT; Drexel University College of Medicine, Philadelphia, PA; Columbia University Medical Center, New York, NY; University of Alabama at Birmingham, Birmingham, AL; Loyola University Medical Center, Maywood, IL; Brooke Army Medical Center, San Antonio, TX; University of Kansas Medical Center, Kansas City, KS; UCLA Medical Center, Los Angeles, CA; The University of Texas Health Science Center at San Antonio, San Antonio, TX; Dallas VA Medical Center, Dallas, TX; William Jennings Bryan Dorn VA Medical Center, Columbia, SC; University of Louisville Hospital, Louisville, KY; Banner Good Samaritan Medical Center, Phoenix, AZ; VA Pittsburgh Healthcare System, Pittsburgh, PA; Duke University, Durham, NC; Baylor All Saints Medical Center, Fort Worth, TX.

\section{Disclosure}

The authors report no conflicts of interest in this work.

\section{References}

1. Gines P, Schrier RW. Renal failure in cirrhosis. $N$ Engl J Med. 2009;361:1279-1290.

2. Salerno F, Gerbes A, Gines P, Wong F, Arroyo V. Diagnosis, prevention and treatment of hepatorenal syndrome in cirrhosis. Gut. 2007;56: 1310-1318.

3. Cardenas A, Gines P. Hepatorenal syndrome. Clin Liver Dis. 2006; 10:371-385.
4. Benza RL, Miller DP, Gomberg-Maitland M, et al. Predicting survival in pulmonary arterial hypertension: insights from the Registry to Evaluate Early and Long-Term Pulmonary Arterial Hypertension Disease Management (REVEAL). Circulation. 2010;122:164-172.

5. Sarela AI, Mihaimeed FM, Batten JJ, Davidson BR, Mathie RT. Hepatic and splanchnic nitric oxide activity in patients with cirrhosis. Gut. 1999; 44:749-753.

6. Tristani FE, Cohn JN. Systemic and renal hemodynamics in oliguric hepatic failure: effect of volume expansion. J Clin Invest. 1967;46: 1894-1906.

7. Newby DE, Jalan R, Masumori S, Hayes PC, Boon NA, Webb DJ. Peripheral vascular tone in patients with cirrhosis: role of the reninangiotensin and sympathetic nervous systems. Cardiovasc Res. 1998;38: 221-228.

8. Gines A, Escorsell A, Gines P, et al. Incidence, predictive factors, and prognosis of the hepatorenal syndrome in cirrhosis with ascites. Gastroenterology. 1993;105:229-236.

9. Alessandria C, Ozdogan O, Guevara M, et al. MELD score and clinical type predict prognosis in hepatorenal syndrome: relevance to liver transplantation. Hepatology. 2005;41:1282-1289.

10. Marrero J, Martinez FJ, Hyzy R. Advances in critical care hepatology. Am J Respir Crit Care Med. 2003;168:1421-1426.

11. Muir AJ, Sanders LL, Heneghan MA, Kuo PC, Wilkinson WE, Provenzale D. An examination of factors predicting prioritization for liver transplantation. Liver Transpl. 2002;8:957-961.

12. Merion RM, Schaubel DE, Dykstra DM, Freeman RB, Port FK, Wolfe RA. The survival benefit of liver transplantation. Am JTransplant. 2005;5:307-313.

13. Rimola A, Gavaler JS, Schade RR, el-Lankany S, Starzl TE, Van Thiel DH. Effects of renal impairment on liver transplantation. Gastroenterology. 1987;93:148-156.

14. Gonwa TA, Klintmalm GB, Levy M, Jennings LS, Goldstein RM, Husberg BS. Impact of pretransplant renal function on survival after liver transplantation. Transplantation. 1995;59:361-365.

15. Sanyal AJ, Boyer T, Garcia-Tsao G, et al. A randomized, prospective, double-blind, placebo-controlled trial of terlipressin for type 1 hepatorenal syndrome. Gastroenterology. 2008;134:1360-1368.

16. Solanki P, Chawla A, Garg R, Gupta R, Jain M, Sarin SK. Beneficial effects of terlipressin in hepatorenal syndrome: a prospective, randomized placebo-controlled clinical trial. J Gastroenterol Hepatol. 2003;18:152-156.

17. Fabrizi F, Dixit V, Messa P, Martin P. Terlipressin for hepatorenal syndrome: a meta-analysis of randomized trials. Int J Artif Organs. 2009;32:133-140.

18. Martin-Llahi M, Pepin MN, Guevara M, et al. Terlipressin and albumin vs albumin in patients with cirrhosis and hepatorenal syndrome: a randomized study. Gastroenterology. 2008;134: 1352-1359.

19. International Conference on Harmonisation Working Group. ICH Harmonised Tripartite Guideline: Guideline for Good Clinical Practice E6 (R1). International Conference on Harmonisation of Technical Requirements for Registration of Pharmaceuticals for Human Use; June 10, 1996; Washington, DC. International Conference on Harmonisation. Available at: http://www.ich.org/fileadmin/Public_Web_Site/ ICH_Products/Guidelines/Efficacy/E6_R1/Step4/E6_R1_Guideline. pdf. Accessed April 19, 2012.

20. Pugh RN, Murray-Lyon IM, Dawson JL, Pietroni MC, Williams R. Transection of the oesophagus for bleeding oesophageal varices. $\mathrm{Br} J$ Surg. 1973;60:646-649.

21. Ferenci P, Lockwood A, Mullen K, Tarter R, Weissenborn K, Blei AT. Hepatic encephalopathy - definition, nomenclature, diagnosis, and quantification: final report of the working party at the 11th World Congresses of Gastroenterology, Vienna, 1998. Hepatology. 2002;35:716-721.

22. Kamath PS, Kim WR. The model for end-stage liver disease (MELD). Hepatology. 2007;45:797-805.

23. Gines P, Guevara M, Arroyo V, Rodes J. Hepatorenal syndrome. Lancet. 2003;362:1819-1827. 
24. Boyer TD, Sanyal AJ, Garcia-Tsao G, et al. Impact of liver transplantation on the survival of patients treated for hepatorenal syndrome type 1. Liver Transpl. 2011;17:1328-1332.

25. Huang CT, Lin HC, Chang SC, Lee WC. Pre-operative risk factors predict post-operative respiratory failure after liver transplantation. PLoS One. 2011;6:e22689.

26. Nair S, Verma S, Thuluvath PJ. Pretransplant renal function predicts survival in patients undergoing orthotopic liver transplantation. Hepatology. 2002;35:1179-1185.

27. Saner FH, Cicinnati VR, Sotiropoulos G, Beckebaum S. Strategies to prevent or reduce acute and chronic kidney injury in liver transplantation. Liver Int. 2012;32:179-188.

28. Restuccia T, Ortega R, Guevara M, et al. Effects of treatment of hepatorenal syndrome before transplantation on posttransplantation outcome. A case-control study. J Hepatol. 2004;40:140-146.

29. Gluud LL, Christensen K, Christensen E, Krag A. Systematic review of randomized trials on vasoconstrictor drugs for hepatorenal syndrome. Hepatology. 2010;51:576-584.

30. Neri S, Pulvirenti D, Malaguarnera M, et al. Terlipressin and albumin in patients with cirrhosis and type I hepatorenal syndrome. Dig Dis Sci. 2008;53:830-835.

31. Kanagarajan K, Marraffa JM, Bouchard NC, Krishnan P, Hoffman RS, Stork CM. The use of vasopressin in the setting of recalcitrant hypotension due to calcium channel blocker overdose. Clin Toxicol (Phila). 2007;45:56-59.

32. Meyer S, McGuire W, Gottschling S, Mohammed SG, Gortner L. The role of vasopressin and terlipressin in catecholamine-resistant shock and cardio-circulatory arrest in children: review of the literature. Wien Med Wochenschr. 2011;161:192-203.

33. Rehberg S, Ertmer C, Kohler G, et al. Role of arginine vasopressin and terlipressin as first-line vasopressor agents in fulminant ovine septic shock. Intensive Care Med. 2009;35:1286-1296.

34. Bernadich C, Bandi JC, Melin P, Bosch J. Effects of F-180, a new selective vasoconstrictor peptide, compared with terlipressin and vasopressin on systemic and splanchnic hemodynamics in a rat model of portal hypertension. Hepatology. 1998;27:351-356.
35. Laporte R, Kohan A, Heitzmann J, et al. Pharmacological characterization of FE 202158, a novel, potent, selective, and short-acting peptidic vasopressin V1a receptor full agonist for the treatment of vasodilatory hypotension. J Pharmacol Exp Ther. 2011;337:786-796.

36. Forsling ML, Aziz LA, Miller M, Davies R, Donovan B. Conversion of triglycylvasopressin to lysine-vasopressin in man. $J$ Endocrinol. 1980;85:237-244.

37. Nilsson G, Lindblom P, Ohlin M, Berling R, Vernersson E. Pharmacokinetics of terlipressin after single iv doses to healthy volunteers Drugs Exp Clin Res. 1990;16:307-314.

38. Rajekar H, Chawla Y. Terlipressin in hepatorenal syndrome: Evidence for present indications. J Gastroenterol Hepatol. 2011;26 Suppl 1: 109-114.

39. Sagi SV, Mittal S, Kasturi KS, Sood GK. Terlipressin therapy for reversal of type 1 hepatorenal syndrome: a meta-analysis of randomized controlled trials. J Gastroenterol Hepatol. 2010;25:880-885.

40. Boyer TD, Sanyal AJ, Garcia-Tsao G, et al. Predictors of response to terlipressin plus albumin in hepatorenal syndrome (HRS) type 1: relationship of serum creatinine to hemodynamics. J Hepatol. 2011;55: 315-321.

41. Nazar A, Pereira GH, Guevara M, et al. Predictors of response to therapy with terlipressin and albumin in patients with cirrhosis and type 1 hepatorenal syndrome. Hepatology. 2010;51:219-226.

42. Liu J, Wess J. Different single receptor domains determine the distinct $\mathrm{G}$ protein coupling profiles of members of the vasopressin receptor family. J Biol Chem. 1996;271:8772-8778.

43. Walum H, Westberg L, Henningsson S, et al. Genetic variation in the vasopressin receptor 1a gene (AVPR1 A) associates with pairbonding behavior in humans. Proc Natl Acad Sci U S A. 2008;105 14153-14156.
Open Access Journal of Clinical Trials

\section{Publish your work in this journal}

The Open Access Journal of Clinical Trials is an international, peerreviewed, open access journal publishing original research, reports, editorials, reviews and commentaries on all aspects of clinical tria design, management, legal, ethical and regulatory issues, case record form design, data collection, quality assurance and data auditing

\section{Dovepress}

methodologies. The manuscript management system is completely online and includes a very quick and fair peer-review system, which is all easy to use. Visit http://www.dovepress.com/testimonials.php to read real quotes from published authors. 